\title{
Letter about M\&N Paper of Russo et al. 2017
}

\author{
Ugo Carraro* \\ IRCCS Fondazione Ospedale San Camillo, Venezia, Italy
}

Dear Sir,

I read with interest but considerable concern a recent paper published in Muscle and Nerve by Pinheiro-Dardis et al. (Electrical stimulation delays reinnervation in denervated rat muscle doi: 10.1002/ mus.25589). [1] I do not find that the data within the paper justify the claim of the title.

The typescript reports an experiment over two weeks during which the experimental animals were anesthetized daily for more than one hour, but it is not reported if the body weight in all groups increased over the two week study. The authors only state that the body weight of the experimental groups was 'around 25\% less' than the normal control group that did not received daily anaesthesia. Unless the groups were set up with a considerable difference in mean body weight, the implication is that the growth rate of the experimental rats was reduced. Since the study focused on nerve regrowth and recovery of muscle function, this is a major point. In particular the apparently detrimental effect of electrical stimulation, which of course represents additional muscle activity, might be due to the relative lack of availability of nutrients for muscle growth. However the mean muscle weights, though reported to have been measured in the study, are not reported. It is not surprising that the experimental groups had a lower muscle mass than the $\mathrm{N}$ group given that the body mass was $25 \%$ lower and that the muscles had been denervated. The statement that the denervated-stimulated group at $15 \mathrm{~d}$ differed from the other groups, is meaningless without the mean values of all the groups.

Furthermore, presence of nerve injury was assessed by microscopy of the nerve, but it is not reported what criterion was selected for deciding that there was no injury as mentioned under 'nerve morphology' in Material and Methods and how many animals were excluded based on it.

The assessment of excitability data is problematic. As the authors state, both chronaxie and rheobase tend to be increased with denervation, but the authors saw a decline in rheobase. They stress a greater increase in chronaxie in the denervated/stimulated group but in the denervated group the chronaxie showed no sign of returning to control values after 14 days so it is remarkable that the authors claim any effect on reinnervation.

Finally, the authors misuse the reference to the papers of Willand et al. [3-5] that makes the discussion misleading: they state that those papers show that electrical stimulation 'exacerbated' muscle atrophy and force loss whereas even the titles of the papers show that their authors demonstrated that electrical stimulation enhances functional recovery and reduces muscle atrophy. Furthermore, I would like to stress that FES of denervated muscles may recover them from atrophy and degeneration [9-11].

It is important that readers are aware of these critical points to avoid the wrong conclusions on the value of electrical stimulation of denervated/reinnervating muscles.

\section{References}

1. Pinheiro-Dardis CM, Erbereli BT, Gigo-Benato D, Castro PA, Russo TL (2017) Electrical stimulation delays reinnervation in denervated rat muscle. Muscle Nerve 2017 Mar 1. doi: 10.1002/mus.25589. [Crossref]

2. Willand MP, Holmes M, Bain JR, Fahnestock M, De Bruin H (2013) Electrical muscle stimulation after immediate nerve repair reduces muscle atrophy without affecting reinnervation. Muscle Nerve 48: 219-225. [Crossref]

3. Willand MP (2015) Electrical Stimulation Enhances Reinnervation After Nerve Injury Eur J Transl Myol 25: 243-248. [Crossref]

4. Willand MP, Chiang CD, Zhang JJ, Kemp SW, Borschel GH (2015) Daily Electrical Muscle Stimulation Enhances Functional Recovery Following Nerve Transection and Repair in Rats. Neurorehabil Neural Repair. 29: 690-700. [Crossref]

5. Willand MP, Rosa E, Michalski B, Zhang JJ, Gordon T et al. (2016) Electrical muscle stimulation elevates intramuscular BDNF and GDNF mRNA following peripheral nerve injury and repair in rats. Neuroscience. 334: 93-104. [Crossref]

6. Kern H, Boncompagni S, Rossini K et al. (2004) Long-term denervation in human causes degeneration of both contractile and excitation- contraction coupling apparatus, wich is reversibile by functional electrical stimulation (FES). A role for myofiber regeneration? J Neuropathol Exp Neurol 63: 919-931.

7. Boncompagni S, Kern H, Rossini K, Hofer C, Mayr W et al. (2007) Structura differentiation of skeletal muscle fibers in the absence of innervation in humans. Proc Natl Acad Sci U S A 104: 19339-19344. [Crossref]

8. Kern H, Carraro U, Adami $\mathrm{N}$ et al. (2010) Home-based functional electrical stimulation rescues permanently denervated muscles in paraplegic patients with complete lower motor neuron lesion. Neurorehabil Neural Repair 24: 709-721. [Crossref]

9. Gargiulo P, Reynisson PJ, Helgason B, Kern H, Mayr W, et al. (2011) Muscle, tendons, and bone: structural changes during denervation and FES treatment. Neurol Res 33 : 750-758. [Crossref]

10. Carraro U, Kern H, Gava P, Hofer C, Loefler S et al. (2015) Biology of Muscle Atrophy and of its Recovery by FES in Aging and Mobility Impairments: Roots and By-Products. Eur J Transl Myol 25: 221-230. [Crossref]

11. Kern H, Hofer C, Loefler S et al. (2017) Atrophy, ultra-structural disorders, severe atrophy and degeneration of denervated human muscle in SCI and Aging. Implications for their recovery by Functional Electrical Stimulation, updated 2017. Neurol Res 2017 Apr 13:1-7. doi: 10.1080/01616412.2017.1314906. [Epub ahead of print].
Copyright: (C)2017 Carraro U. This is an open-access article distributed under the terms of the Creative Commons Attribution License, which permits unrestricted use, distribution, and reproduction in any medium, provided the original author and source are credited.
Correspondence to: Professor Ugo Carraro, IRCCS Fondazione Ospedale San Camillo, Venezia, Italy, E-mail: ugo.carraro@ospedalesancamillo.net

Received: July 20, 2017; Accepted: July 27, 2017; Published: July 31, 2017 